\title{
ANTHOCYANINS SYNTHESIS IN POTATO (Solanum tuberosum L.): GENETIC MARKERS FOR SMART BREEDING (review)
}

\section{K.V. STRYGINA ${ }^{1}$, E.K. KHLESTKINA1, 2}

\author{
${ }^{1}$ Federal Research Center Institute of Cytology and Genetics SB RAS, Federal Agency of Scientific Organizations, 10, \\ prosp. Akademika Lavrent'eva, Novosibirsk, 630090 Russia, e-mail khlest@bionet.nsc.ru; \\ ${ }^{2}$ Novosibirsk State University, 2, ul. Pirogova, Novosibirsk, 630090 Russia \\ ORCID: Khlestkina E.K. orcid.org/0000-0002-8470-8254 \\ The authors declare no conflict of interests \\ Acknowledgements: \\ Supported by Russian Science Foundation (grant № 16-16-04073) \\ Received November 5, 2016
}

\section{Abstract}

Potato may have anthocyanin-colored tuber skin, tuber flesh, flowers, leaves, stems and eyes. Anthocyanins protect photosynthetic apparatus of plant cell, scavenge free radicals under stress conditions, increase efficiency of phosphorus and nitrogen uptake, possess osmoregulatory function, antimicrobial activity and have a number of other useful properties. Anthocyanins are also known for their health benefit: diabetes type II and cardiovascular diseases protection, anti-inflammatory effect, etc. Thus, anthocyanins are important for adaptation of plants to unfavorable environment conditions as well as for nutritional value when they are taken with food. Since potato Solanum tuberosum L. is one of the main crop species, possibility to increase anthocyanin content in tuber flesh is important. Anthocyanin concentration in pigmented tuber flesh is similar to that in blueberries, blackberries, cranberries and red grapes. It is important that cooking as well as long storage of potato tubers doesn't affect anthocyanin content. Coloration traits (red or purple tuber flesh) are included in ongoing breeding programs. Therefore, development of tools (convenient diagnostic PCR-markers for anthocyanin biosynthesis genes) for accelerated and efficient selection is of importance. The goal of the current review is to summarize information on the genes regulating anthocyanin biosynthesis in potato and assess possibility of development of diagnostic marker for prediction of tuber flesh color before tuber formation. Anthocyanin biosynthesis takes place in cytosol with the help of enzymes CHS, CHI, DFR, F3H, F3' H, F3'5' H and ANS, after that anthocyanins are transported to vacuoles. Activation of biosynthesis is controlled by MBW complex consisting of transcription factors MYB, bHLH and WD40. This complex activates transcription of structural genes encoding the enzymes mentioned above. A number of MYB-encoding genes are identified in potato, among them StAN1 related with anthocyanin biosynthesis. This gene corresponds to the $D$ locus previously revealed with genetic dissection approach and mapped to chromosome 10. The genes encoding bHLH (StJAF13 and $S t b H L H 1)$ and WD40 (StWD40) have been revealed only by their homology with similar genes of other plant species, but not by genetic dissection, probably because they have no allelic diversity. Thus, the main gene determining high variability of potato by the coloration traits is StAN1. Its allelic variants are described and shown to be related with anthocyanin synthesis efficiency. The StAN1 alleles can be easily distinguished by PCR fragments lengths, what allows constructing convenient diagnostic markers for selection. In some cases, the lack of anthocyanins is due to mutation of a structural gene. This was described in the literature for the $R$ locus encoding DFR enzyme. Mutation of other structural gene, StF3'5'H (locus $P$ ), just partially disrupts anthocyanin synthesis, not effecting red pigments, but blue and purple only. This makes the $S t F 3^{\prime} 5^{\prime} H$ an attractive target for markerassisted identification of genotypes with different tuber flesh color - purple or red. Thus, there are two main targets for breeding anthocyanin-colored potato - StAN1 and StF3'5'H.

Keywords: Solanum tuberosum, potato, marker-assisted selection, anthocyanins, stress tolerance, nutrition value, genes, diagnostic markers

Anthocyanins are plant pigments of a large family of phenolic compounds, the flavonoids. In potato (Solanum tuberosum L.) the anthocyanins can cause the coloration of the tuber peel (pink, red, red, blue and dark purple), the pulp of the tuber (red and violet, solid or patchy, of different saturation), eyes 
and flowers (red and blue, unequal intensity), leaves and stems (from weak to very intense) [1].

Color of the stem and leaves is worthy of attention, as these are adaptive signs. Thus, anthocyanins participate in the protection of the photosynthetic apparatus and cell membrane, neutralize free radicals, protect against their adverse effects, increase the efficiency of phosphorus and nitrogen assimilation, enhance the osmoregulatory function, reduce the freezing temperature of cell contents, and provide chelation and sequestration of heavy metal ions. These properties in different combination allow plants to adapt to unfavorable conditions, e.g. excessive UV radiation, drought, extreme temperatures, soil salinity, phosphorus and nitrogen deficiency, toxic effects of herbicides and heavy metals [2-5]. Also anthocyanins have antimicrobial activity [6, 7]. For example, their significant contribution to the resistance of potatoes to wet rot is shown: on sections of tubers with purple pulp, the lesion area was on average $28.6 \%$ less than in potatoes with yellow pulp [8]. In plant tissues in the infection zone, there is a rapid oxidation of phenolic compounds, including anthocyanins, followed by lignification, suberinization and programmed cell death [9].

The anthocyanin content in tubers and, especially, in their pulp is of the greatest importance in potato as a food crop. It is known that anthocyanins coming from plant foods can prevent cardiovascular diseases, type II diabetes, arthritis, obesity, vision pathologies, various cancers and neurodegenerative diseases, have anti-inflammatory effects [10-15]. The molecular mechanisms underlying these properties are not completely clear. For some polyphenol compounds, possible participation in the regulation of gene activity, signaling, exposure to cell receptors and blood proteins involved in the inflammatory response (acute phase proteins) [16-18] is indicated. Antioxidant properties of anthocyanins are also discussed. In particular, it is not clear whether they are able to neutralize free radicals in vivo in the same way as in vitro (the interaction efficiency in vitro of anthocyanins against various reactive oxygen species is almost 4 times higher than that of ascorbic acid and $\alpha$-tocopherol) [3]. The therapeutic (hypoglycemic) effect of anthocyanins is described, which is associated with the suppression of genes involved in the synthesis of aliphatic acids and triacylglycerol, as well as inhibition of pancreatic amylase and intestinal $\alpha$-glucosidase [19-21].

Tubers with a purple pulp are a rich source of anthocyanins, in particular of their acylated derivatives $(22,23)$. Anthocyanidin derivatives, the petunidin, pelargonidin, peonidine and malvidin, are detected in potato cultivars [24] (Fig. 1). The content of anthocyanins in colored tubers is comparable to that in blueberries, blackberries, cranberries and red grapes in which these compounds are synthesized in the largest amount $[25,26]$. In addition, flavonoids mainly accumulate in potato peel rich in substances that are of pharmacological interest [27, 28]. It is important that, after cooking, the amount of anthocyanins either decreases insignificantly, or does not change [29, 30]. The same happens when storing potatoes [31].

The properties of anthocyanins stimulate involvement of the potato in breeding programs for a high content of these pigments in the red and purple pulp of the tuber [32, 33]. Increasing the effectiveness of such programs is now associated with the development of DNA markers for target anthocyanin biosynthesis genes based on the use of PCR analysis. The use of DNA markers during the early breeding steps (instead of evaluating biochemical parameters after the manifestation of the trait) allows the selection only of the forms with the desired genes and reduces time and costs for creation of cultivars [34].

The purpose of this review is to analyze data on the genes that regulate the biosynthesis of anthocyanins in potato plants and to evaluate the possibility 
of developing DNA markers that predict the nature of the pulp color in early ontogeny prior to the onset of tuber formation.

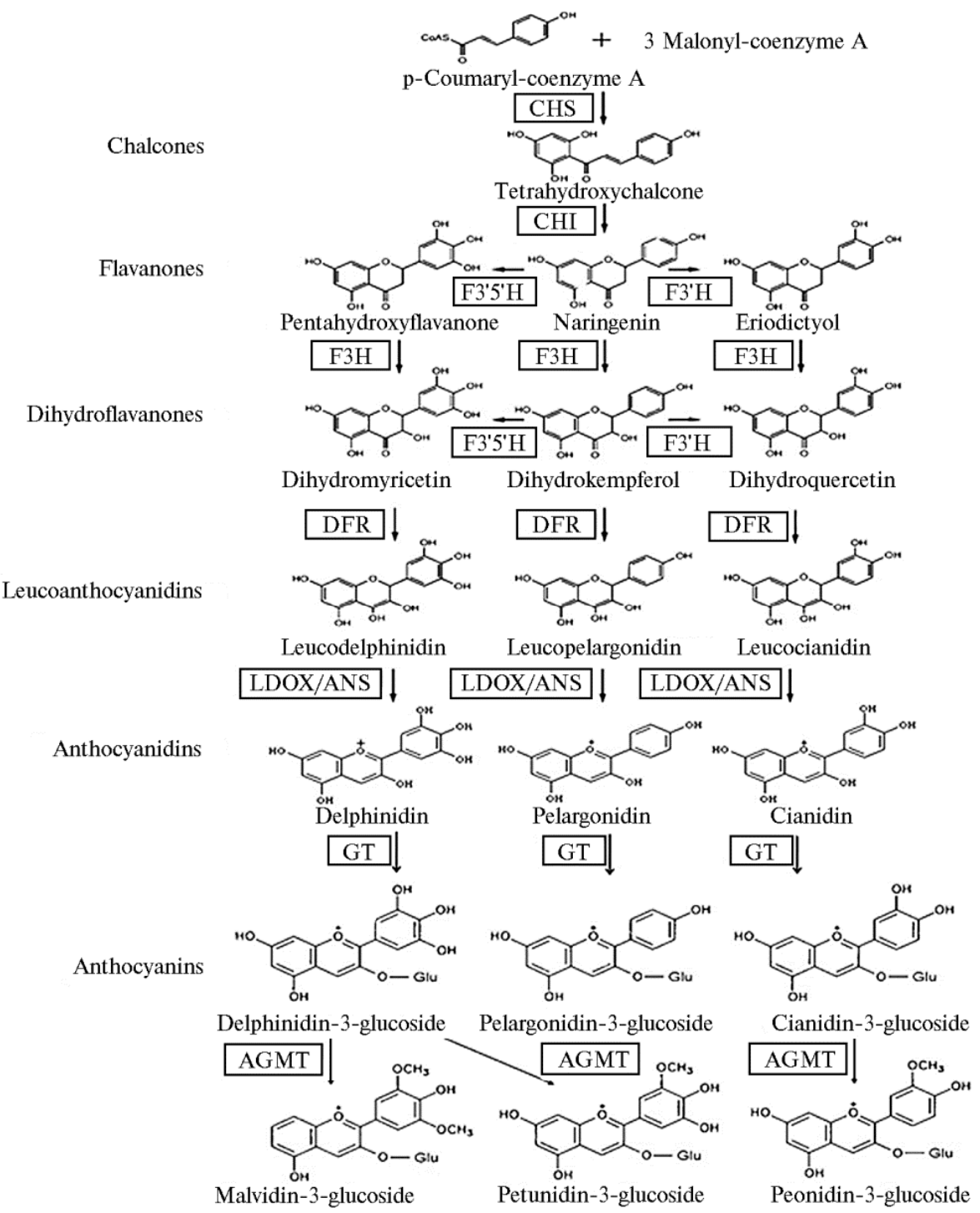

Fig. 1. The biosynthesis scheme for the most common anthocyanin pigments (cited in 51): CHS chalcone synthase, $\mathrm{CHI}$ - chalcone-flavanon isomerase, F3H - flavanon-3-hydroxylase, F3' $\mathrm{H}$ flavonoid-3'-hydroxylase, F3'5'H - flavonoid-3',5'-hydroxylase, DFR - dihydroflavonol-4reductase, LDOX/ANS - leucoanthocyanidin dioxygenase/anthocyanidin synthase, GT - glycosyltransferase, AGMT - anthocyanidin glucoside 3'-O-methyltransferase.

Genetic regulation of anthocyanins biosynthesis. The enzymes involved in biosynthesis of anthocyanins and their precursors in higher plants are CHS (chalcone synthase), CHI (chalcone flavanone isomerase), DFR (dihydroflavonol-4-reductase), F3H (flavanon-3-hydroxylase), F3' H (flavonoid-3'-hydroxylase), F3'5'H (flavonoid-3',5'-hydroxylase), LDOX/ANS (leucoanthocyanidin dioxygenase/anthocyanidin synthase) (see Fig. 1). After synthesis in the cytosol, the phenolic compounds are transported to cell vacuoles [35]. In all plant species analyzed to date, tissue-specific accumulation of anthocyanins is associated with the structural gene expression of abovementioned enzymes regulated by three types of transcription factors (TF), i.e. domain MYB, the basic domain "helix-loop-helix" (bHLH) and WD-repeats (repeats of two amino acid 
residues, tryptophan and aspartic acid, WD) [36-39].

The synthesis of anthocyanins in the periderm of tubers of $S$. tuberosum tetraploid potato was shown to be controlled by loci $P$ (purple) and $R$ (red) mapped in chromosomes 11 and 2, respectively (40-44). In this case, the locus $P$ is epistatic to $R$ [40]. Later it was found that $P$ and $R$ are genes encoding the enzymes of flavonoid biosynthesis (F3'5'H and DFR) [45-48] (see Fig. 1). Now the molecular mechanisms underlying the nature of $P$ and $R$ inheritance are clear. Red pigments, the derivatives of pelargonidin, can be synthesized in plant cells with functional CHS, CHI, F3H, DFR and ANS enzymes (see Fig. 1), but not in gene $R$ mutant with defective DFR. Synthesis of violet pigments, the derivatives of petunidine and its precursor delphinidin, necessitates the enzyme $\mathrm{F}^{\prime} 5^{\prime} \mathrm{H}$ encoded by $P$ locus (see Fig. 1). When it is functional, the precursors of the red pigment are used in the synthesis of delphinidin and petunidin thus switching biosynthesis pathway in a manner which leads to epistasis revealed by genetic analysis. Using DNA analysis, the location of genes encoding other enzymes was determined. $C H S$ and $C H I$ were found on chromosome 5, ANS - on chromosome 8 [47, 49, 50].

In addition to structural genes, $S$. tuberosum has loci corresponding to regulatory genes. Locus $D$ (developer; in diploid potatoe $S$. rybinii Juz. \& Bukasov designated as $I-$ inhibitor) located on chromosome 10 encodes the transcription factor R2R3 MYB, which has a high similarity with the product of the previously isolated petunia (Petunia hybrida) regulatory gene - PhAN2 [27, 40, 52, 53]. Additionally to MYB-like TF, orthologs of the petunia genes encoding bHLH, PhJAF13 and PhAN1, are found in S. tuberosum on chromosomes 8 and 9, respectively [47, 49, 50].

Regulatory MYB factors. MYB family is one of the most common groups of TF described in plants. Proteins from this family have two specific domains - conservative N-terminal DNA-binding domain of about 50 amino acids and a non-conservative $\mathrm{C}$-terminal region responsible for regulation of the target gene expression [54]. The common characteristic for all MYB factors is the presence of one to four incomplete MYB repeats that can function together or separately in binding DNA and protein-protein interactions, respectively. By the number of repetitions in the MYB domain, this family can be divided into four classes: 1R, R2R3, 3R and 4R [55]. R2R3 MYB family is the largest group of genes that encode TF in plants. They play an important role in regulation of catalytic enzymes, including the biosynthesis of anthocyanins [54, 56].

Because of tetraploidy and heterozygosity of cultivated potato $S$. tuberosum, the combination of MYB proteins is more complex than in diploid potatoes. In recent years, much attention has been paid to molecular mechanisms and genes controlling the biosynthesis of anthocyanins or their accumulation in potato tubers [57-62], and all researches emphasized the role of gene StAN1ANTHOCYANIN1 (GenBank accession number JQ418343) (Fig. 2, A) in controlling the expression of structural genes involved in the biosynthesis of anthocyanins and other phenylpropanoids, especially in the periderm and pulp of pigmented tubers [57, 58, 63]. Besides, StAN1 is one of the key genes responsible for differences in the biosynthesis of anthocyanins not only in tubers, but in potato leaves [59]. The StAN1 sequence resembles the petunia gene PhAN2, so this locus was originally named similarly, but was later renamed [57]. StAN1 is not only an important regulator of anthocyanins biosynthesis, but also a key factor in the synthesis of other phenylpropanoids in tubers. It is noted that the expression of the StAN1 gene correlates with accumulation of flavonoids in potatoes under response to drought [63].

Sucrose (see below) is involved in regulation of StAN1 gene activity [58]. 
The role of this compound in modulating the transcriptional and post-translational regulation of many genes associated with pigmentation is well known [64]. Sucrose may enhance the synthesis of anthocyanins, but is not capable of causing changes in anthocyanin deficient mutants $[65,66]$.

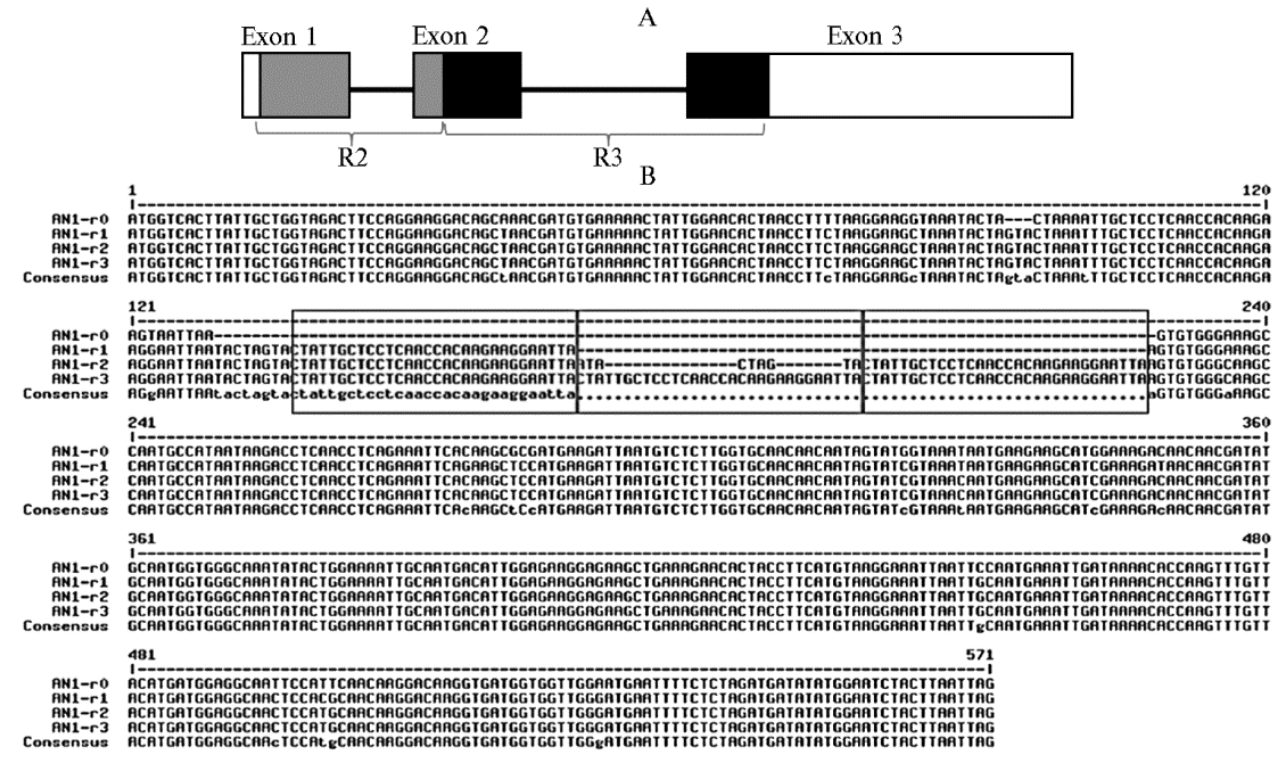

Fig. 2. Regulatory factor StAN1 gene organization: A - exon-intron structure of StAN1 [57], the DNA-binding domains of R2 and R3 MYB are noted; B - nucleotide sequences of the exon 3 in different alleles $(S t A N 1-r 0,-r 1,-r 2,-r 3)$ of the StAN1 gene (rectangles indicate motifs r, the number of which affects the efficiency of anthocyanin synthesis).

High intraspecific variability of the StAN1 gene is noted [59, 61], with mutations detected both in the coding and non-coding regions (i.e. in the intron 2 and the exon 3 of the gene). Some mutations lead to loss of important amino acids, necessary for interaction with DNA. Most mutations of StAN1 are either silencing, or are in introns. First, StAN1 ${ }^{816}$ (AY841128) and StAN1777 (AY841130) were found in StAN1 [57], and it was noted that they are found only in potato cultivars with pigmentation of tuber periderm. Allel StAN1 ${ }^{816}$ is $39 \mathrm{bp}$ longer than the $S t A N 1^{777}$ in exon 3, and 82 bp shorter in the intron 2, so in the PCR analysis, it is easy to distinguish $S t A N 1^{816}$ from $S t A N 1^{777}$ by 43 bp shorter amplification fragments. Later, other alleles were detected, StAN1-r0 (KM822778), StAN1-r1 (KM822779), StAN1-r3 (KM822780) [61], and it turned out that the r-motif (see Fig. 2) is a duplication of $30 \mathrm{bp}$ long region (CTATTGCTCCTCAACCACAAGAAGGAATTA, 10 amino acid residues - TIAPQPQEGI) in the exon 3. Thus, the alleles are named depending on whether they have these repeats and how numerous the repeats are. The allele StAN1-r1 is defined by us as StAN1777, and $S t A N 1^{816}$ is proposed to be designated as $S t A N 1-r 2$, because in its sequence there are two perfect (non-degenerate) r-repetitions (see Fig. 2, B). It is assumed that the presence of one $r$-motif in the exon 3 is optimal for the activation of anthocyanin synthesis, and the absence of r-motif or its excess (three replicates) decreases the functionality of the protein [61]. The manifestation of the allele with two replicates of the r-motif has not yet been evaluated.

StAN1 is expressed in both normal and pigmented tubers, and the expression level of the gene is not proportional to the amount of flavonoids in the plant. Interestingly, the most StAN1 transcripts detected in potato cultivars with nonpigmented peel and pulp had a truncated 3 '-end. The product of such truncated mRNA is probably unable to activate the biosynthesis of anthocyanins, which may indicate the importance of r-motif as a functional element of StAN1 [61)]. There- 
fore, PCR analysis can be developed to accurately identify and map allelic variants of the gene along the DNA amplification fragments. These markers can be used in breeding to select the most effective variant of the regulatory gene StAN1.

Allelic differences of StAN1 affect not only exons and introns. The number of elements associated with sucrose (SURE, sucrose-responsive element) and methyl jasmonate (MeJa, methyl jasmonate-responsive elements) varies in promoters of genes responsible for pigmentation. According to R.S. Payyavula et al. [58], alleles of the StAN1 gene that cause accumulation of anthocyanins in tubers contain up to six SURE sequences and up to five MeJa motifs in the promoters. In another study, the presence of the SINE retrotransposone in the promoter was shown to be associated with anthocyanin pigmentation of leaves. In genotypes with green leaves (without anthocyanin pigmentation), this element does not appear in the StAN1 promoter [59]. The presence of mobile elements modifies the expression of genes encoding MYB [67-71], hence further study of the modification effect of SINE on the StAN1 promoter functions is necessary.

Since some parts (or tissues) of the potato plant (phelloderm, tuber periderm, eyes, leaves, stems, etc.) can be pigmented independently, it has been suggested that locus $D$ contains two (or more) tandem duplication of $M Y B$ gene, at that the phenotype modifications are associated with changes in the sequences of different $M Y B$ genes [57, 59]. Indeed, by the homology with StAN1, another gene was found, first designated as StAN3, then renamed to StAN2, and also having the synonym StMYBA1 [57-61]. The predicted amino acid sequence for StMYBA1 with high similarity corresponds to a protein of the R2R3 MYB class (66\% identity to StAN1) [58]. StMYBA1 was assumed to be a copy of StAN1, lost functionality and turned into a pseudogene, as full transcripts of StMYBA1 could not be detected [57]. Later it was found out that expression of this gene can be connected with synthesis of anthocyanins in potato tubers, i.e. two allelic variants, StMYBA1-1 which can be expressed in all tissues irrespective of their pigmentation, and StMYBA1-2, active only in violet tubers [61], have been identified. Expression in StMYBA1 is significantly lower than that of StAN1, and its strict correlation with accumulation of polyphenols in tubers is not evident. Probably, StMYBA1 regulates the transcription of not only anthocyanin biosynthesis genes [57, 63].

In Potato Genome Sequencing Consortium (PGSC) database (http://solanaceae.plantbiology.msu.edu/pgsc_download.shtml), other nucleotide sequences for MYB factors conservative in the R2 and R3 domains [58] were identified. One of them, $M Y B 12 B$, is weakly expressed in the pulp of tubers with a clear inverse correlation between transcriptional activity and the amount of phenylpropanoids. Expression of $M Y B 12 B$ in the remaining organs and tissues of potatoes was not studied. Perhaps this is a truncated transcript of one of the $M Y B$ genes found in potatoes.

By homology to AtMYB113 which positively regulates metabolism of phenylpropanoid in Arabidopsis thaliana [72], StMYB113 [61] was detected and three functionally different gene variants, StMYBA113-1, StMYBA113-2 and StMYBA113-3, were identified. StMYBA113-1 is expressed in both pigmented and nonpigmented potato tissues, while StMYBA113-3 was active only in the violet peel, but has a shortened protein product due to a $130 \mathrm{bp}$ deletion detected in the cDNA and causing the stop codon at the amino acid 9 position. Compared to other alleles, StMYBA113-2, which is expressed only in the red peel, has several deletions leading to amino acid substitutions.

Thus, among MYB-like transcription factors, TF, encoded by the StAN1 gene for which a clear correlation between the functionality of the alleles and the variability in $\mathrm{C}$-end of the protein is revealed, plays a key role in the regulation of 
anthocyanin synthesis. Additionally, StMYBA1 and StMYB113 associated with the production of anthocyanins may also have the potential for selection.

Regulatory bHLH factors. As it was noted, various R2R3 MYBs regulate the biosynthesis of phenylpropanoids, at that some of the proteins interact to the bHLH factors [36]. bHLH family form the second largest class of TF in plants. The bHLH domain of about 60 amino acids in length is highly conservative. It consists of two functionally distinct regions. The N-end contains 1317 amino acids and binds to the E-box (enhancer box, present in some promoter regions in the eukaryotic DNA sequence of CANNTG, where $\mathrm{N}$ can be any nucleotide). The HLH consists of two amphipathic $\alpha$-helices which mainly contain hydrophobic amino acids and are connected by a loop variable in length. Proteins containing the HLH motif often form homo- or heterodimers with other bHLH proteins, which is necessary for DNA recognition. The interaction of bHLH to R2R3 MYB involved in formation of transcriptional complexes with the promoters of anthocyanin biosynthesis genes seems to be rather important. Thus, the MYB transcription factor ( $P p-1$ gene) in the absence of bHLH encoded by the $P p 3$ gene (TaMyc1) is unable to activate the biosynthesis of anthocyanins in the pericarp of wheat weevil [73].

To date, StbHLH1 (JX848660) and StJAF13/StbHLH2 (KP317176) for two bHLH factors which are involved in regulation of the anthocyanin synthesis in tuber and leaves have been identified in $S$. tuberosum [58, 59]. These genes are extremely conservative in domains that determine the structure of the bHLH domain, but in the others are very different, which makes the amino acid sequences only $43 \%$ similar. The combination of StJAF13 and StAN1 functional variants predetermines high activity of anthocyanin biosynthesis structural genes and the presence of a pigment in the leaves and tubers peel [59]. The obtained data indicate a complementary interaction of the regulatory factors StAN1 and StJAF13, as a result of which anthocyanins are synthesized [59]. The level of anthocyanin expression is determined by StAN1 gene, whereas transcription of StJAF13 and also StbHLH1, another gene encoding bHLH, does not correlate to accumulation of phenylpropanoids, including anthocyanins, in the pigmented tissues [58, 61].

Mutant variants of the StJAF13 gene, on the basis of which diagnostic markers could be created, are not yet known in potato. For the StbHLH1 gene, five allelic variants are described and their functional role in certain tissues is assumed [61], but this information is subject to additional experimental verification.

Regulatory WD40 factor. The biosynthesis of anthocyanins is usually regulated by the MBW complex formed by the transcription factors MYB, bHLH and WD40. The first 200 amino acids of bHLH are required for interaction with MYB, and the next 200 residues are involved in interaction with WD40 [74]. WD proteins contain four to eight degenerate tandem repeats and interact with other proteins via the repeats [75].

So far, StWD40 is known to be the only found tetraploid potato gene, the expression of which correlates to the content of phenolic compounds and anthocyanins [58]. Its expression is 3-5 times higher in red and violet tubers. However, this factor alone can not induce the synthesis of phenolic compounds. That is, WD40 is necessary, but not sufficient to activate the anthocyanin pathway. StWD40 gene mutations in potato have not yet been described.

Structural gene $F 3^{\prime} 5^{\prime} H$. Pigmentation of tissues and organs in plants primarily depends on enzymes that directly synthesize polyphenolic molecules. For $S$. tuberosum, the key gene that switches the synthesis from red pigments to blue and violet is $S t F 3^{\prime} 5^{\prime} H$ (see Fig. 1). The cDNA sequence of the $S t F 3^{\prime} 5^{\prime} H$ gene is known (HQ860267). Introduction of the cDNA of this gene as a 
transgene changes the color of the peel of potato tubers from red to violet [45]. Therefore, when selecting the mutants in gene $S t F 3^{\prime} 5^{\prime} H$, it is possible to change the color of tuber peel or pulp from violet to red which makes such loci potential targets in programs of potato breeding for anthocyanin composition. Based on the sequencing of the functional and mutant alleles of the $S t F 3^{\prime} 5^{\prime} H$, diagnostic markers can be constructed for controlled selection based on the length of fragments in the PCR. StF3'5'H seems to be a promising target not only for marker-oriented selection, but also for obtaining modified forms using modern safe editing methods. So, using the CRISPR/Cas9 [76] system it will be easy to knock out the $S t F 3^{\prime} 5^{\prime} H$ gene to change the color of the tuber peel or pulp from violet to red.

The role of sucrose in the regulation of anthocyanins biosynthesis. The biosynthesis of anthocyanins is usually light-dependent and occurs in the underground parts of the plant as an exception requiring additional mechanisms for activation of the MBW regulatory complex. Such a mechanism in plants is associated with sucrose. Sucrose modulates the regulation of the expression of many genes involved in the synthesis of pigments at the transcriptional and post-translational levels [64]. Sucrose is not only a source of carbon for phenylpropanoid metabolism (through the products of its hydrolysis), but also an anthocyanin biosynthesis regulator [65]. In red and purple tubers, the content of sucrose and glucose is higher than in white or yellow tubers. Sucrose significantly stimulates the expression of StAN1, StbHLH1 and StWD4O, enhancing the phenylpropanoid potato metabolism [58].

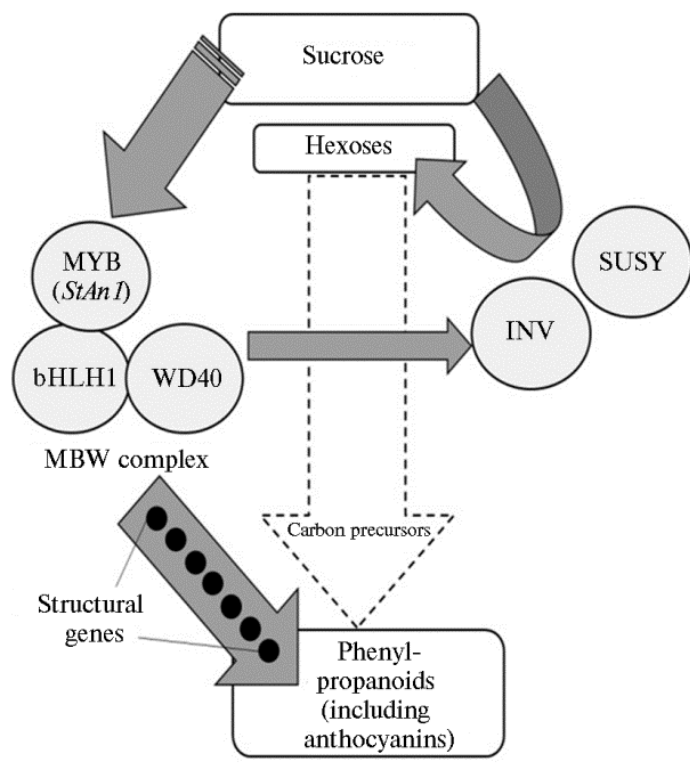

Fig. 3. The proposed model for the interaction of MBW transcription factors complex, sucrose and its metabolism factors in the regulation of the synthesis of phenylpropanoids in potato tubers [58]. Sucrose stimulates the expression of the StAN1 gene encoding the MYB transcription factor; MYB together with bHLH1 and WD40 (transcription factors of the MBW complex) triggers the synthesis of phenylpropanoids (via activation of the transcription of the structural genes of biosynthetic enzymes) and regulates the expression of the sucrose enzyme (SuSy - sucrose synthase, Inv - invertase) genes. The effect of SUSY and INV factors leads to a decrease in the content of sucrose and accumulation of hexoses, the derivatives of which serve as a substrate for the synthesis of phenylpropanoids.

The presence of SURE elements in the StAN1 promoter is consistent with the assumption of regulating its expression with sucrose:

in potato with purple and red tubers, there are six SURE elements, whereas white and yellow ones are characterized by the presence of a single SURE element [58]. Sucrose treatment with potato seedlings significantly increases the expression of StAN1 and StbHLH1, and to a lesser extent StWD40. There is a hypothesis about a possible regulatory loop: sucrose activates the expression of the factors that make up the MBW complex, and that reduces the content of sucrose by inducing the enzymes of its hydrolysis with the release of hexoses, the decay products of which serve as precursors in the synthesis of phenylpropanoids (Fig. 3) [58].

So, the synthesis of anthocyanins in potato tubers is activated by the 
MBW complex which is formed by transcription factors MYB, bHLH and WD40. The genes encoding these factors are StAN1, StJAF13 (and StbHLH1), and also StWD40. Unlike the conservative genes of transcription factors bHLH and WD40, the StAN1 gene encoding MYB is characterized by a considerable variability. Its alleles are described and their relationship with the efficiency of anthocyanin biosynthesis is shown. StAN1 alleles can be differentiated by the length of specific PCR products. The formation of red or violet pigments depends on flavonoid-3', $5^{\prime}$-hydroxylase $\left(\mathrm{F}^{\prime} 5^{\prime} \mathrm{H}\right)$. Plants with a normal function of MBW regulatory complex and all structural genes of anthocyanins biosynthesis (including $S t F 3^{\prime} 5^{\prime} H$ ) will produce a purple pigment, whereas the presence of the mutant StF3'5'H gene leads to red pigment production. Thus, StAN1 and $S t F 3^{\prime} 5^{\prime} H$ are currently considered as the main target genes in the selection of anthocyanin synthesizing potato plants.

\section{R E F E R E N C E S}

1. Metodicheskie ukazaniya po podderzhaniyu $i$ izucheniyu mirovoi kollektsii kartofelya /Pod redaktsiei S.D. Kiru [Potato world collection: recommendations on specimen maintenance and preservation. S.D. Kiru (ed.)]. St. Petersburg, 2010 (in Russ.).

2. Chalker-S ott L. Environmental significance of anthocyanins in plant stress responses. Photochem. Photobiol., 1999, 70(1): 1-9 (doi: 10.1111/j.1751-1097.1999.tb01944.x).

3. Gould K.S. Nature's Swiss army knife: the diverse protective roles of anthocyanins in leaves. BioMed Research International, 2004, 2004(5): 314-320 (doi: 10.1155/S1110724304406147).

4. Hale K.L., Tufan H. A., Pickering I.J., George G.N., Te rry N., Pilon M., Pilon-S mits E.A. Anthocyanins facilitate tungsten accumulation in Brassica. Physiologia Plantarum, 2002, 116(3): 351-358 (doi: 10.1034/j.1399-3054.2002.1160310.x).

5. Kh lest ki n a E. The adaptive role of flavonoids: emphasis on cereals. Cereal Res. Commun., 2013, 41(2): 185-198 (doi: 10.1556/CRC.2013.0004).

6. Cisowska A., Wojnicz D., Hendrich A.B. Anthocyanins as antimicrobial agents of natural plant origin. Nat. Prod. Commun., 2011, 6(1): 149-156.

7. We n H., Ka ng J., Li D., We n W., Y ang F., H u H., Li u C. Antifungal activities of anthocyanins from purple sweet potato in the presence of food preservatives. Food Sci. Biotechnol., 2016, 25(1): 165-171 (doi: 10.1007/s10068-016-0025-7).

8. Wege ne r C.B., J a ns e n G. Soft-rot resistance of coloured potato cultivars (Solanum tuberosum L.): the role of anthocyanins. Potato Res., 2007, 50(1): 31-44 (doi: 10.1007/s11540-007-9027-4).

9. B e c k ma n C.H. Phenolic-storing cells: keys to programmed cell death and periderm formation in wilt disease resistance and in general defense responses in plants. Physiological and Molecular Plant Pathology, 2000, 57(3): 101-110 (doi: 10.1006/pmpp.2000.0287).

10. Cassidy A., O'Re illy É.J., Kay C., S a m pson L., Franz M., Forman J.P., $\mathrm{Cu}$ r h a n G., Ri m m E.B. Habitual intake of flavonoid subclasses and incident hypertension in adults. Am. J. Clin. Nutr., 2011, 93(2): 338-347 (doi: 10.3945/ajcn.110.006783).

11. How a rd B.V., K rit chevsky D., Nutrition Committee. Phytochemicals and cardiovascular disease a statement for healthcare professionals from the American heart association. Circulation, 1997, 95(11): 2591-2593 (doi: 10.1161/01.CIR.95.11.2591).

12. Hui C., Bi Y., Xia op in Y., Lon Y., Chunye C., Mantian M., Wenhua L. Anticancer activities of an anthocyanin-rich extract from black rice against breast cancer cells in vitro and in vivo. Nutrition and Cancer, 2010, 62(8): 1128-1136 (doi: 10.1080/01635581.2010.494821).

13. S a n c ho R.A.S., Pas t o r e G.M. Evaluation of the effects of anthocyanins in type 2 diabetes. Food Res. Int., 2012, 46(1): 378-386.

14. Li la M.A. Anthocyanins and human health: an in vitro investigative approach. BioMed Research International, 2004, 2004(5): 306-313 (doi: 10.1155/S111072430440401X).

15. Wang H., N a i r M.G., Strasburg G.M., Chang Y.C., B o o r e n A.M., G r a y J.I., DeWitt D.L. Antioxidant and antiinflammatory activities of anthocyanins and their aglycon, cyanidin, from tart cherries. J. Nat. Prod., 1999, 62(2): 294-296 (doi: 10.1021/np980501m).

16. Willi a m s R.J., S p e n c e r J.P., R i c e-Evan s C. Flavonoids: antioxidants or signalling molecules? Free Radical Bio. Med., 2004, 36(7): 838-849 (doi: 10.1016/j.freeradbiomed.2004.01.001).

17. Aggarwal B.B., S h is hodi a S. Molecular targets of dietary agents for prevention and therapy of cancer. Biochem. Pharmacol., 2006, 71(10): 1397-1421 (doi: 10.1016/j.bcp.2006.02.009).

18. Virgili F., M a ri no M. Regulation of cellular signals from nutritional molecules: a specific role for phytochemicals, beyond antioxidant activity. Free Radical Bio. Med., 2008, 45(9): 12051216 (doi: 10.1016/j.freeradbiomed.2008.08.001).

19. Tsuda T., Horio F., Uchida K., Aoki H., Osawa T. Dietary cyanidin 3-O- $\beta$-D- 
glucoside-rich purple corn color prevents obesity and ameliorates hyperglycemia in mice. $J$. Nutr., 2003, 133(7): 2125-2130.

20. S h ob a n a S., S r e e r a ma Y.N., Malle shi N.G. Composition and enzyme inhibitory properties of finger millet (Eleusine coracana L.) seed coat phenolics: Mode of inhibition of $\alpha$-glucosidase and pancreatic amylase. Food Chem., 2009, 115(4): 1268-1273 (doi: 10.1016/j.foodchem.2009.01.042).

21. Tadera K., Minami Y., Takamatsu K., Matsuoka T. Inhibition of $\alpha$-glucosidase and $\alpha$ amylase by flavonoids. J. Nutr. Sci. Vitaminol., 2006, 52(2): 149-153 (doi: 10.3177/jnsv.52.149).

22. Rodrigue z-S a o n a L.E., Wrols t a d R.E., P e re i r a C. Glycoalkaloid content and anthocyanin stability to alkaline treatment of red-fleshed potato extracts. J. Food Sci., 1999, 64(3): 445-450 (doi: 10.1111/j.1365-2621.1999.tb15060.x).

23. Fos se n T., A n d e rs e n Ø.M. Anthocyanins from tubers and shoots of the purple potato, Solanum tuberosum. The Journal of Horticultural Science and Biotechnology, 2000, 75(3): 360-363 (doi: 10.1080/14620316.2000.11511251).

24. Eich horn S., Winterhalter P. Anthocyanins from pigmented potato (Solanum tuberosum L.) varieties. Food Res. Int., 2005, 38(8): 943-948 (doi:10.1016/j.foodres.2005.03.011).

25. Andre C.M., Oufir M., Guignard C., Hoffmann L., H a u s man J.F., Eve r s D., L a r o n d e 11 e Y. Antioxidant profiling of native Andean potato tubers (Solanum tuberosum L.) reveals cultivars with high levels of $\beta$-carotene, $\alpha$-tocopherol, chlorogenic acid, and petanin. J. Agr. Food Chem., 2007, 55(26): 10839-10849 (doi: 10.1021/jf0726583).

26. K a 1 i t a D., J a y a $\mathrm{n} \mathrm{t}$ y S.S. Comparison of polyphenol content and antioxidant capacity of colored potato tubers, pomegranate and blueberries. Journal of Food Processing and Technology, 2014, 5: 358 (doi: 10.4172/2157-7110.1000358).

27. Le w is C.E., W a $1 \mathrm{k}$ e r J.R., La n c a s t e $\mathrm{r}$ J.E., S u t t o n K.H. Determination of anthocyanins, flavonoids and phenolic acids in potatoes. I: Coloured cultivars of Solanum tuberosum L. J. Sci. Food Agr., 1998, 77(1): 45-57 (doi: 10.1002/(SICI)1097-0010(199805)77:1<45::AIDJSFA1>3.0.CO;2-S).

28. S c h i e b e r A., S a $1 \mathrm{~d}$ a n a M.A. Potato peels: a source of nutritionally and pharmacologically interesting compounds - a review. Food, 2009, 3(2): 23-29.

29. Mulinacci N., I eri F., Giaccherini C., Innocenti M., And renelli L., C a nova G., S a r a c chi M., C a siragh i M.C. Effect of cooking on the anthocyanins, phenolic acids, glycoalkaloids, and resistant starch content in two pigmented cultivars of Solanum tuberosum L. J. Agr. Food Chem., 2008, 56(24): 11830-11837 (doi: 10.1021/jf801521e).

30. L e mos M.A., A li y u M.M. H u nge rford G. Influence of cooking on the levels of bioactive compounds in Purple Majesty potato observed via chemical and spectroscopic means. Food Chem., 2015, 173: 462-467 (doi: 10.1016/j.foodchem.2014.10.064).

31. Jansen G., Flamme W. Coloured potatoes (Solanum tuberosum L.) - anthocyanin content and tuber quality. Genet. Resour. Crop Ev., 2006, 53(7): 1321-1331 (doi: 10.1007/s10722-005-3880-2).

32. B row n C.R., Wrolst ad R., Durst R., Y ang C.P., C le vid e n c e B. Breeding studies in potatoes containing high concentrations of anthocyanins. Am. J. Potato Res., 2003, 80(4): 241-249 (doi: 10.1007/BF02855360).

33. B r ow n C.R., Culle y D., Y a n g C.P., D u r s t R., W rolst a d R. Variation of anthocyanin and carotenoid contents and associated antioxidant values in potato breeding lines. $J$. Am. Soc. Hortic. Sci., 2005, 130(2): 174-180.

34. K h le s t k i n E.K., S h u m y i V.K., K o l c ha nov N.A. Dostizheniya nauki i tekhniki $A P K$, 2016, 30(10): 5-8 (in Russ.).

35. K o e s R., Verwe i j W., Q u a t t r o c c h i o F. Flavonoids: a colorful model for the regulation and evolution of biochemical pathways. Trends Plant Sci., 2005, 10(5): 236-242 (doi: 10.1016/j.tplants.2005.03.002).

36. G r o t e w o ld E. Plant metabolic diversity: a regulatory perspective. Trends Plant Sci., 2005, 10(2): 57-62 (doi: 10.1016/j.tplants.2004.12.009).

37. C o n e K.C., B u r r F.A., B u r r B. Molecular analysis of the maize anthocyanin regulatory locus C1. PNAS, 1986, 83(24): 9631-9635.

38. Ludwig S.R., Habe ra L.F., D e 11 a porta S.L., W e s s le r S.R. Lc, a member of the maize $R$ gene family responsible for tissue-specific anthocyanin production, encodes a protein similar to transcriptional activators and contains the myc-homology region. PNAS, 1989, 86(18): 7092-7096.

39. Qu at troc chio F., Wing J.F., V a K., M o l J.N., K o e s R. Analysis of bHLH and MYB domain proteins: species-specific regulatory differences are caused by divergent evolution of target anthocyanin genes. Plant J., 1998, 13(4): 475-488 (doi: 10.1046/j.1365-313X.1998.00046.x).

40. Dodds K., Lo ng D.H. The inheritance of colour in diploid potatoes. J. Genet., 1955, 53(1): 136-149 (doi: 10.1007/BF02981517).

41. va n E ck H.J., J a c obs J.M., van Dijk J., S tieke ma W.J., J a c obse n E. Identification and mapping of three flower colour loci of potato ( $S$. tuberosum L.) by RFLP analysis. Theor. Appl. Genet., 1993, 86(2-3): 295-300 (doi: 10.1007/BF00222091). 
42. va n E c k H.J., J a c obs J.M., va n d e n B e rg P.M., S ti e k e m a W.J., J a c ob s e $n$ E. The inheritance of anthocyanin pigmentation in potato (Solanum tuberosum L.) and mapping of tuber skin colour loci using RFLPs. Heredity, 1994, 73: 410-421 (doi: 10.1038/hdy.1994.189).

43. G e b hardt C., Rit te r E., D ebener T., S c hach t s chabel U., Wa lk e m e i e r B., Uhrig H., S a la mini F. RFLP analysis and linkage mapping in Solanum tuberosum. Theor. Appl. Genet., 1989, 78(1): 65-75 (doi: 10.1007/BF00299755).

44. J a c obs J.M.E., van Eck H.J., A rens P., Verkerk-Bakker B., t e Linte 1 H e k k e rt B., B astia a s s e n H.J.M., El- Kharbotly A., P e rei ra A., J a c ob s e $\mathrm{n}$ E., S t i e k e m a W.J. A genetic map of potato (Solanum tuberosum) integrating molecular markers, including transposons, and classical markers. Theor. Appl. Genet., 1995, 91(2): 289300 (doi: 10.1007/BF00220891).

45. J ung C.S., Griffiths H.M., D e Jong D.M., Cheng S., B od is M., D e Jong W.S. The potato $P$ locus codes for flavonoid 3',5'-hydroxylase. Theor. Appl. Genet., 2005, 110(2): 269-275 (doi: 10.1007/s00122-004-1829-z).

46. Zhang Y., Cheng S., De Jong D., Griffiths H., Halitschke R., De $\mathrm{J}$ o $\mathrm{ng} \mathrm{W}$. The potato $R$ locus codes for dihydroflavonol 4-reductase. Theor. Appl. Genet., 2009, 119(5): 931-937 (doi: 10.1007/s00122-009-1100-8).

47. D e J ong W.S., E a n n e t t a N.T., D e J o n g D.M., B o d is M. Candidate gene analysis of anthocyanin pigmentation loci in the Solanaceae. Theor. Appl. Genet., 2004, 108(3): 423432 (doi: 10.1007/s00122-003-1455-1).

48. D e J ong W.S., De Jong D.M., D e Jong H., K a la zich J., B o d is M. An allele of dihydroflavonol 4-reductase associated with the ability to produce red anthocyanin pigments in potato (Solanum tuberosum L.). Theor. Appl. Genet., 2003, 107(8): 1375-1383 (doi: 10.1007/s00122-003-1395-9).

49. Z h a n g Y., J u ng C.S., D e J o ng W.S. Genetic analysis of pigmented tuber flesh in potato. Theor. Appl. Genet., 2009, 119(1): 143-150 (doi: 10.1007/s00122-009-1024-3).

50. S p e $1 \mathrm{t}$ C., Q u a t t r o c c h i o F., M o l J.N., Koes R. anthocyanin 1 of petunia encodes a basic helix-loop-helix protein that directly activates transcription of structural anthocyanin genes. Plant Cell, 2000, 12(9): 1619-1631 (doi: 10.1105/tpc.12.9.1619).

51. Grotew old E. The genetics and biochemistry of floral pigments. Annu. Rev. Plant Biol., 2006, 57: 761-780 (doi: 10.1146/annurev.arplant.57.032905.105248).

52. D e $\mathrm{J}$ o $\mathrm{ng} \mathrm{H}$. Inheritance of anthocyanin pigmentation in the cultivated potato: a critical review. Am. Potato J., 1991, 68(9): 585-593 (doi: 10.1007/BF02853712).

53. Stushnoff C., Ducreux L. J., H a ncock R.D., Hedley P.E., Holm D.G., M c D o ugall G.J., M c Nicol J.W., Morris J., Morris W.L., S u ngurtas J.A., Verra 11 S.R., Z u b e r T., T a y lor M.A. Flavonoid profiling and transcriptome analysis reveals new gene-metabolite correlations in tubers of Solanum tuberosum L. J. Exp. Bot., 2010, 61 (4): 1225-1238 (doi: 10.1093/jxb/erp394).

54. F e lle r A., M a c h e mer K., B ra u E.L., G rote w o ld E. Evolutionary and comparative analysis of MYB and bHLH plant transcription factors. Plant J., 2011, 66(1): 94-116 (doi: 10.1111/j.1365-313X.2010.04459.x).

55. Dubos C., Stracke R., Grotewold E., We is shaar B., Marti n C., Le p i n i e c L. MYB transcription factors in Arabidopsis. Trends Plant Sci., 2010, 15(10): 573-581 (doi: 10.1016/j.tplants.2010.06.005).

56. A 11 a n A.C., H e $11 \mathrm{e} n \mathrm{~s}$ R.P., L a i ng W.A. MYB transcription factors that colour our fruit. Trends Plant Sci., 2008, 13(3): 99-102 (doi: 10.1016/j.tplants.2007.11.012).

57. J ung C.S., Griffiths H.M., D e Jong D.M., Cheng S., B od is M., Ki m T.S., D e J o ng W.S. The potato developer $(D)$ locus encodes an R2R3 MYB transcription factor that regulates expression of multiple anthocyanin structural genes in tuber skin. Theor. Appl. Genet., 2009, 120(1): 45-57 (doi: 10.1007/s00122-009-1158-3).

58. $\mathrm{P}$ a y y a vula R.S., $\mathrm{S}$ i $\mathrm{ngh}$ R.K., $\mathrm{N}$ a v a $\mathrm{r}$ e D.A. Transcription factors, sucrose, and sucrose metabolic genes interact to regulate potato phenylpropanoid metabolism. J. Exp. Bot., 2013, 64(16): 5115-5131 (doi: 10.1093/jxb/ert303).

59. D'Amelia V., Aversano R., B a te 11 i G., C a ruso I., C a s te 11 a no More no M., C a st ro-S anz A.B., $\mathrm{C}$ hi a i e s e P., F a s a no C., P a $10 \mathrm{mb}$ a F., C a r p u t o D. High $A N 1$ variability and interaction with basic helix-loop-helix co-factors related to anthocyanin biosynthesis in potato leaves. Plant J., 2014, 80(3): 527-540 (doi: 10.1111/tpj.12653).

60. T a i H.H., G o y e r C., M u r p h y A.M. Potato MYB and bHLH transcription factors associated with anthocyanin intensity and common scab resistance. Botany, 2013, 91(10): 722-730 (doi: 10.1139/cjb-2012-0025).

61. Li u Y., Lin-Wang K., Espley R.V., Wang L., Yang H., Yu B., D a re A., Varkonyi-Gas i c E., W a ng J., Z hang J., W a ng D., A 11 a n A.C. Functional diversification of the potato R2R3 MYB anthocyanin activators AN1, MYBA1, and MYB113 and their interaction with basic helix-loop-helix cofactors. J. Exp. Bot., 2016, 67(8): 2159-2176 (doi: 10.1093/jxb/erw014). 
62. Liu Y., Lin-Wang K., Deng C., Warran B., Wang L., Yu B., Yang H., W a ng D., Espley R.V., Z hang J., W ang D., A 11 a n A.C. Comparative transcriptome analysis of white and purple potato to identify genes involved in anthocyanin biosynthesis. PloS ONE, 2015, 10(6): e0129148 (doi: 10.1371/journal.pone.0129148).

63. André C.M., Schafleitner R., Legay S., L e fèv re I., Ali a g a C.A.A., N o m b e r to G., Hoffmann a L., H a u s m a n a J., La rond e 11 e b Y., Evers D. Gene expression changes related to the production of phenolic compounds in potato tubers grown under drought stress. Phytochemistry, 2009, 70(9): 1107-1116 (doi: 10.1016/j.phytochem.2009.07.008).

64. K o c h K.E. Carbohydrate-modulated gene expression in plants. Annu. Rev. Plant Biol., 1996, 47(1): 509-540 (doi: 10.1146/annurev.arplant.47.1.509).

65. Teng S., Keurentjes J., B entsink L., Koornneef M., S meekens S. Sucrose-specific induction of anthocyanin biosynthesis in Arabidopsis requires the MYB75/PAP1 gene. Plant Physiol., 2005, 139(4): 1840-1852 (doi: 10.1104/pp.105.066688).

66. S olfa ne 11 i C., P ogg i A., L oret i E., Alp i A., P e r a t a P. Sucrose-specific induction of the anthocyanin biosynthetic pathway in Arabidopsis. Plant Physiol., 2006, 140(2): $637-$ 646 (doi: 10.1104/pp.104.900185).

67. Kobayashi S., Goto-Yamamoto N., Hi rochik a H. Retrotransposon-induced mutations in grape skin color. Science, 2004, 304(5673): 982-982 (doi: 10.1126/science.1095011).

68. Walker A.R., Lee E., Bogs J., McDavid D.A., Thomas M.R., Robinson S.P. White grapes arose through the mutation of two similar and adjacent regulatory genes. Plant J., 2007, 49(5): 772-785 (doi: 10.1111/j.1365-313X.2006.02997.x).

69. Telias A., Lin-Wang K., Stevenson D.E., Cooney J.M., Hellens R.P., Allan A.C., Hoover E.E., Bradeen J.M. Apple skin patterning is associated with differential expression of MYB10. BMC Plant Biol., 2011, 11: 93 (doi: 10.1186/1471-2229-11-93).

70. Butelli E., Licciardello C., Zhang Y., Liu J., Mackay S., Bailey P., Reforgiato-Recupero G., Martin C. Retrotransposons control fruit-specific, colddependent accumulation of anthocyanins in blood oranges. Plant Cell, 2012, 24(3): 1242-1255 (doi: 10.1105/tpc.111.095232).

71. Lisch D. How important are transposons for plant evolution? Nat. Rev. Genet., 2013, 14(1): 49-61 (doi: 10.1038/nrg3374).

72. Borevitz J.O., Xia Y., Blount J., Dixon R.A., Lamb C. Activation tagging identifies a conserved MYB regulator of phenylpropanoid biosynthesis. Plant Cell, 2000, 12(12): 2383-2393 (doi: 10.1105/tpc.12.12.2383).

73. Gordeeva E.I., Shoeva O.Y., Khlestkina E.K. Marker-assisted development of bread wheat near-isogenic lines carrying various combinations of purple pericarp $(P p)$ alleles. Euphytica, 2015, 203(2): 469-476 (doi: 10.1007/s10681-014-1317-8).

74. Pattanaik S., Kong Q., Zaitlin D., Werkman J.R., Xie C.H., Patra B., Yuan L. Isolation and functional characterization of a floral tissue-specific R2R3 MYB regulator from tobacco. Planta, 2010, 231(5): 1061-1076 (doi: 10.1007/s00425-010-1108-y).

75. Neer E.J., Schmidt C.J., Nambudripad R., Smith T.F. The ancient regulatory-protein family of WD-repeat proteins. Nature, 1994, 371(6495): 297-300 (doi: 10.1038/371297a0).

76. Kh lest ki na E.K., Shumny i V.K. Genetika, 2016, 52(7): $774-787$ (doi: 10.7868/S0016675816070055) (in Russ.). 